\title{
Cost-effectiveness of guideline-endorsed treatments for low back pain: a systematic review
}

\author{
Chung-Wei Christine Lin - Marion Haas • \\ Chris G. Maher · Luciana A. C. Machado • \\ Maurits W. van Tulder
}

Received: 12 December 2010/Accepted: 19 December 2010/Published online: 13 January 2011

(C) The Author(s) 2011. This article is published with open access at Springerlink.com

\begin{abstract}
Healthcare costs for low back pain (LBP) are increasing rapidly. Hence, it is important to provide treatments that are effective and cost-effective. The purpose of this systematic review was to investigate the cost-effectiveness of guideline-endorsed treatments for LBP. We searched nine clinical and economic electronic databases and the reference list of relevant systematic reviews and included studies for eligible studies. Economic evaluations conducted alongside randomised controlled trials investigating treatments for LBP endorsed by the guideline of the American College of Physicians and the American Pain Society were included. Two independent reviewers screened search results and extracted data. Data extracted included the type and perspective of the economic evaluation, the treatment comparators, and the relative cost-
\end{abstract}

C.-W. C. Lin $(\varangle) \cdot$ C. G. Maher

The George Institute for Global Health and Sydney Medical

School, The University of Sydney, PO Box M201,

Missenden Rd, Sydney, NSW 2050, Australia

e-mail: clin@george.org.au

\section{Haas}

Centre for Health Economics Research and Evaluation, The University of Technology, PO Box 123, Broadway, Sydney, NSW 2007, Australia

\section{A. C. Machado}

Departamento de Fisioterapia e Terapia Ocupacional, Universidade Federal de Minas Gerais, Av Antônio Carlos 6627,

Pampulha, Belo Horizonte, MG 31270-901, Brazil

\section{W. van Tulder}

Department of Health Sciences and the EMGO Institute for Health and Care Research, Faculty of Earth and Life Sciences, VU University, de Boelelaan 1085,

1081 HV Amsterdam, The Netherlands effectiveness of the treatment comparators. Twenty-six studies were included. Most studies found that interdisciplinary rehabilitation, exercise, acupuncture, spinal manipulation or cognitive-behavioural therapy were costeffective in people with sub-acute or chronic LBP. Massage alone was unlikely to be cost-effective. There were inconsistent results on the cost-effectiveness of advice, insufficient evidence on spinal manipulation for people with acute LBP, and no evidence on the cost-effectiveness of medications, yoga or relaxation. This review found evidence supporting the cost-effectiveness of the guidelineendorsed treatments of interdisciplinary rehabilitation, exercise, acupuncture, spinal manipulation and cognitivebehavioural therapy for sub-acute or chronic LBP. There is little or inconsistent evidence for other treatments endorsed in the guideline.

Keywords Low back pain - Cost-effectiveness analysis . Systematic review

\section{Introduction}

Low back pain (LBP) is a common health condition which affects most adults at some point during their lifetime [1]. For most patients in primary care, the source of symptoms cannot be specified and the patient receives the label nonspecific $L B P$ [2]. The exceptions are those with back pain associated with radiculopathy or spinal stenosis [3] and the rare patients whose LBP can be attributed to a disease or condition such as fracture, tumour or infection [4]. Recently, the American College of Physicians and the American Pain Society published a joint clinical guideline which recommended the following treatments for nonspecific LBP [3]: 
- Provide evidence-based information on prognosis, advise to remain active, provide information about effective self-care options (referred to as advice for the rest of the paper)

- In addition, consider the use of medications with proven benefits

- For patients who do not improve, consider the addition of spinal manipulation for acute LBP

- For patients who do not improve, consider the addition of interdisciplinary rehabilitation, exercise, acupuncture, massage, spinal manipulation, yoga, cognitivebehavioural therapy or relaxation for sub-acute or chronic LBP

These recommendations are largely in line with other international guidelines [5] and are derived from the vast amount of research regarding the effectiveness of treatments for LBP. For example, the latest issue of The Cochrane Library contains over 30 Cochrane systematic reviews of interventions for LBP [6]. In contrast, until the 2009 British guideline [7], LBP guidelines contained little information on the cost-effectiveness of treatments. This was probably due to the low number of studies available to the developers of the early guidelines. The low number of available studies, together with methodological limitations of the studies and the heterogeneity of the studies, limited any conclusive evidence regarding the cost-effectiveness of interventions for LBP $[8,9]$.

LBP incurs substantial treatment and loss of productivity costs internationally [10]. In the United States, healthcare costs among people with back pain increased by $65 \%$ from 1997 to 2005 , more rapidly than healthcare costs among people without back pain and the overall healthcare costs [11]. Given that the guidelines considered a range of interventions to be effective, the efficiency of treatment will be improved if their relative cost-effectiveness is also considered. As the number of published economic evaluations of interventions for LBP is increasing, it may now be possible to consider evidence of cost-effectiveness when making recommendations about treatment. The purpose of this paper is to investigate the cost-effectiveness of guideline-endorsed treatments for non-specific LBP.

\section{Methods}

Data sources and searches

We conducted a systematic search for economic evaluations (i.e. cost-minimization, cost-effectiveness, cost-utilization or cost-benefit analysis) [12] conducted alongside randomised controlled trials in adults with non-specific LBP. Treatments endorsed in the clinical practice guideline of the American College of Physicians and the American Pain Society were eligible for inclusion (i.e. advice, medication, spinal manipulation for acute LBP, and interdisciplinary rehabilitation, exercise, acupuncture, massage, spinal manipulation, yoga, cognitive-behavioural therapy or relaxation for sub-acute or chronic LBP) [3], except when these treatments were implemented after spinal surgery. To be included, studies had to relate the costs of the interventions to the effects of the interventions, for example by reporting an incremental cost-effectiveness ratio (ICER). There was no language restriction.

We searched six clinical (Medline, EMBASE, CINAHL, Cochrane Central Register of Controlled Trials, PsychINFO and PEDro) and three economic (EconLit, NHS EED and EURONHEED) databases from inception to 1 June 2010. The reference list of relevant systematic reviews and included studies was also searched. Search terms were derived from the search strategies of the Cochrane Back Review Group (http://www.cochrane.iwh.on.ca/pdfs/CBRG_ searchstrat_Sept08.pdf) and the British National Health Services Economic Evaluation Database (NHS EED) (http:// www.york.ac.uk/inst/crd/nhseedfaq02.htm). As an example, the complete search strategy for EMBASE is in Appendix.

Study selection and quality assessment

Each review process (screening, risk of bias assessment and data extraction) was conducted by two independent reviewers, with differences resolved first in discussion, and then (if necessary) by arbitration by a third, independent reviewer. In selecting eligible studies from the search results, first the titles, then abstracts (if available), and then full papers were screened. For the included studies, we used the criteria from the Cochrane Back Review Group $[13,14]$ to assess the risk of bias of the trial design, and the Consensus on Health Economic Criteria (CHEC-list) [15] to assess the methodological quality of the economic evaluation. Studies that scored 6 or more out of a total of 11 on the risk of bias assessment were considered as having a low risk of bias [16]. All publications related to the included studies (e.g. published protocol or clinical outcomes paper) were used to inform the risk of bias assessment and data extraction (see Appendix).

Data extraction, synthesis and analysis

Data were extracted using a customized data extraction sheet, and included: the type and perspective of the economic evaluation, treatment comparators, year/s, country and currency of the study, and results of the relative costeffectiveness of the treatment comparators, which was the primary outcome of interest. As we could not locate an agreed cost-effectiveness threshold for the United States, 
Fig. 1 Flow of study

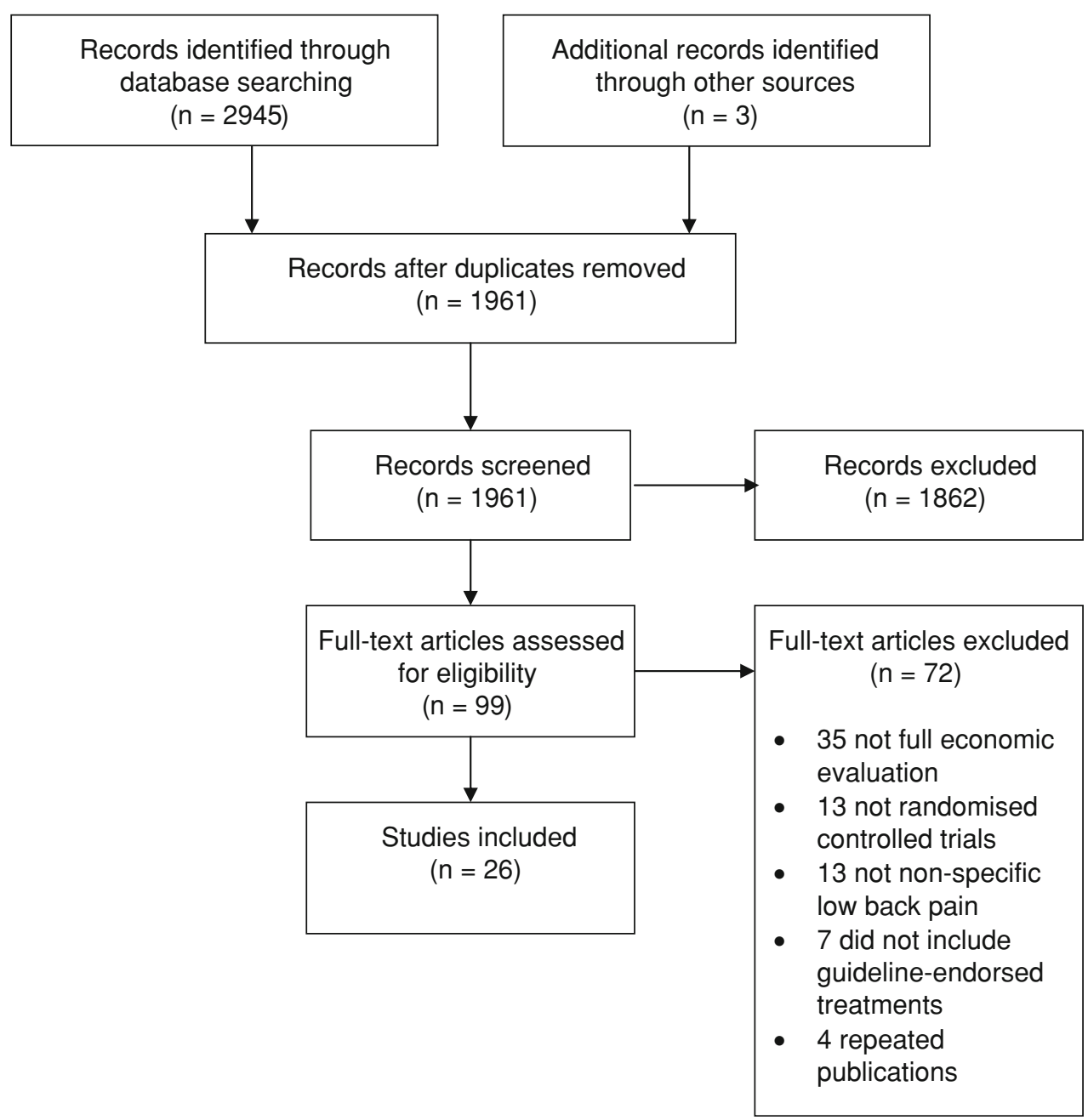

we used the threshold set by the United Kingdom (UK)'s National Institute for Health and Clinical Excellence (NICE) as an indicator for cost-effectiveness [17]. That is, if a treatment had an ICER lower than $£ 20,000-£ 30,000$ per quality-adjusted life-years (QALY) gained, the treatment was deemed as relatively cost-effective compared to an alternate treatment. Where a treatment incurred significantly lower costs and was statistically significantly more effective compared to an alternate treatment, the treatment was considered as "dominant".

\section{Results}

Characteristics of included trials

A total of 26 studies were included after screening the 1961 records found in the search (Fig. 1). Results of two separate studies [18, 19] were reported in one paper [20]. These studies had almost identical design features so are referred to as "Strong et al." in the current paper. For two of the included studies, results of the one- and two-year follow-up were published separately [21-24]. Most studies conducted a cost-effectiveness and/or cost-utility analysis. One study conducted a cost-effectiveness analysis as well as a costbenefit analysis [25]. Four other studies conducted a cost-benefit analysis [26-29] and two studies conducted a cost-minimization analysis $[30,31]$. One study did not clearly state the type of economic evaluation undertaken, but we consider this to be a cost-effectiveness analysis due to the outcomes reported [21, 22].

Most studies recruited participants with at least 4-6 weeks (sub-acute) or greater than 12 weeks (chronic) of LBP. The only exceptions were two studies which recruited participants of a mixed duration of symptoms [30, 32] two which did not specify the duration of symptoms [20], and one which recruited participants sick listed for less than 2 weeks due to LBP [31]. Most studies were conducted in the UK [32-40] or other European countries [21-24, 26-29, 31, 41-45]; three studies were conducted in the United States [20, 30] and two in Canada [25, 46]. All studies were published in English. 
Risk of bias of the trial design

Just over half ( $n=13$ ) of the studies had a low risk of bias. Five of the studies did not report adequate randomization procedures $[19,20,40,41]$, nine did not have adequate allocation concealment [18-20, 23, 24, 29, 31, 39, 41, 44, 46] and none of the studies used assessor blinding (Table 1).

Quality of the economic evaluation

Eleven studies scored 17 or more out of 19 on the CHEClist. Seven studies did not state the economic perspective adopted [21, 22, 29-32, 35, 41]. An incremental costeffectiveness analysis was conducted in all but 2 of the 19 cost-effectiveness or cost-utility studies [23, 24, 41]. Three of the nine studies which had a follow-up period of greater than 1 year did not use discounting [23-25, 30]. Other methodological issues are outlined in the following sections describing the evidence for each treatment (Table 1).

Advice (provide evidence-based information on prognosis, advise to remain active, provide information about effective self-care options)

A total of nine studies were included (Table 2). Six studies compared advice to another treatment [20-22, 27, 38, 46], and three compared adding another treatment to advice with advice alone [23, 24, 26, 36]. Regardless of the comparison and the economic perspective adopted, results regarding the cost-effectiveness of advice were inconsistent across the studies (Table 2). Four studies suggested that advice may be more cost-effective than treatments received in primary care $[21,22,27]$ or a book on back pain care [20], but other studies reported a cost-effectiveness ratio or cost-benefit outcome which favoured adding naturopathic care [46], graded activity [26],or manipulation and stabilizing exercises [23, 24] over advice alone, or physiotherapy over advice [38]. In a study comparing adding manipulation and exercises to advice alone [23, 24], it is unclear why the reported cost-effectiveness ratio was positive for pain but negative for disability, given that costs were identical and the direction of benefits was the same for both outcomes.

There were methodological issues regarding the identification and measurement of costs in five of the eight studies. One study [46] undertook their analysis from the societal perspective, but did not collect the costs of visits to doctors, or secondary or tertiary care. It was unclear why some of the costs reported had a negative value, and their follow-up period (6 months) may be too short to fully capture the economic consequences of sub-acute or chronic LBP. Two studies [20] undertook their analysis from the
Table 1 Risk of bias and methodological quality assessment using the Cochrane Back Review Group criteria [13, 14] and the Consensus Health Economic Criteria (CHEC-list) [15], respectively

\begin{tabular}{|c|c|c|}
\hline & $\begin{array}{l}\text { Risk of bias } \\
\text { score (/11) }\end{array}$ & $\begin{array}{l}\text { CHEC-list } \\
(/ 19)^{\mathrm{a}}\end{array}$ \\
\hline Critchley et al. [33] & 7 & $19(0)$ \\
\hline Goossens et al. [41] & 2 & $14(1)$ \\
\hline Herman et al. [46] & 6 & $17(1)$ \\
\hline Hlobil et al. [26] & 7 & $15(2)$ \\
\hline Hollinghurst et al. [34] & 7 & $17(1)$ \\
\hline Johnson et al. [35] & 7 & $12(1)$ \\
\hline Karjalainen et al. [21, 22] & 8 & $13(0)$ \\
\hline Kominski et al. [30] & 5 & $11(4)$ \\
\hline Lamb et al. [36] & 7 & $15(1)$ \\
\hline Loisel et al. [25] & 5 & $14(0)$ \\
\hline Molde Hagen et al. [27] & 7 & $12(1)$ \\
\hline Niemisto et al. $[23,24]$ & 6 & $16(0)$ \\
\hline Ratcliffe et al. [37] & 5 & $19(0)$ \\
\hline Rivero-Arias et al. [38] & 8 & $18(1)$ \\
\hline Rivero-Arias et al. [39] & 5 & $18(0)$ \\
\hline Schweikert et al. [44] & 5 & $17(1)$ \\
\hline Seferlis et al. [31] & 4 & $7(5)$ \\
\hline Skouen et al. [28] & 5 & $14(1)$ \\
\hline Smeets et al. [42] & 8 & $18(1)$ \\
\hline Strong et al. [20] & 4 & $13(1)$ \\
\hline Torstensen et al. [29] & 6 & $9(2)$ \\
\hline UK BEAM Trial Team [40] & 5 & $17(1)$ \\
\hline Van der Roer et al. [43] & 6 & $18(1)$ \\
\hline Whitehurst et al. [32] & 6 & $17(1)$ \\
\hline Witt et al. [45] & 4 & $13(1)$ \\
\hline
\end{tabular}

Studies with a risk of bias score of 6 or more were considered to have a low risk of bias [16]

a The number of items that were not applicable from the CHEC-list is in parentheses. For example, performing an incremental cost-effectiveness analysis (item 13) was not applicable for cost-minimization or cost-benefit analysis, and discounting (item 14) was not applicable for studies with a follow-up of 1 year or less

health insurer's perspective but excluded the costs of inpatient care. Both studies which conducted a cost-benefit analysis [26, 27] had a 3-year follow-up, but collected only the costs incurred in the first year. One of these studies did not state the methods used to value the costs [27].

In addition, consider the use of medications with proven benefits

No study compared the cost-effectiveness of any medication in managing LBP. This included the first-line recommendation of acetaminophen and non-steroidal anti-inflammatory drugs, more potent analgesics such as opioids or tramadol, and herbal therapies. 
Table 2 The cost-effectiveness of "advice" (provide evidence-based information on prognosis, advise to remain active, provide information about effective self-care options)

\begin{tabular}{|c|c|c|c|}
\hline Study ID & Comparative treatments & $\begin{array}{l}\text { Details of economic } \\
\text { evaluation }\end{array}$ & Results of economic evaluation \\
\hline Herman et al. [46] & $\begin{array}{l}\text { Advice (advice and back booklet) } \\
\text { Naturopathic care (acupuncture, } \\
\text { relaxation) and back booklet }\end{array}$ & $\begin{array}{l}\text { Type: CEA/CUA } \\
\text { Perspective: societal, } \\
\text { employer and patient } \\
\text { Setting: Canada, } 2005 \\
\text { Follow-up: } 6 \text { months }\end{array}$ & $\begin{array}{l}\text { ICER for naturopathic care compared to advice: } \\
\text { QALY (SF-6D) = naturopathic care and back } \\
\text { booklet dominant (i.e. incurred lower costs and } \\
\text { more effective) from societal and patient's } \\
\text { perspective } \\
\text { Absenteeism }=154 \text { (2005 USD) per absentee } \\
\text { day avoided from employer's perspective }\end{array}$ \\
\hline Hlobil et al. [26] & $\begin{array}{l}\text { Advice } \\
\text { Advice plus graded activity using } \\
\text { cognitive-behavioural principles }\end{array}$ & $\begin{array}{l}\text { Type: CBA } \\
\text { Perspective: employer } \\
\text { Setting: The Netherlands, } \\
\text { 1999-2000 } \\
\text { Follow-up: } 1 \text { year for costs, } \\
3 \text { years for other } \\
\text { outcomes }\end{array}$ & $\begin{array}{l}\text { Mean cost benefit }=999 \text { (1999 Euro) favouring } \\
\text { advice plus graded activity ( } 95 \% \text { CI }-1,073 \\
\text { to } 3,115 \text { ) }\end{array}$ \\
\hline Lamb et al. [36] & $\begin{array}{l}\text { Group cognitive behavioural } \\
\text { intervention plus advice } \\
\text { Advice }\end{array}$ & $\begin{array}{l}\text { Type: CEA/CUA } \\
\text { Perspective: healthcare } \\
\text { sector } \\
\text { Setting: United Kingdom, } \\
\text { price year } 2008 \\
\text { Follow-up: } 1 \text { year }\end{array}$ & $\begin{array}{l}\text { ICER for Group cognitive behavioural intervention } \\
\text { plus advice = 1,786 (2008 GBP) per QALY } \\
\text { gained (EQ-5D) }\end{array}$ \\
\hline $\begin{array}{l}\text { Karjalainen et al. } \\
{[21,22]}\end{array}$ & $\begin{array}{l}\text { GP care } \\
\text { GP care plus advice (advice, } \\
\text { education, exercise) }\end{array}$ & $\begin{array}{l}\text { Type and perspective: not } \\
\text { stated } \\
\text { Setting: Finland 1998-2000 } \\
\text { Follow-up: } 24 \text { months }\end{array}$ & $\begin{array}{l}\text { No ICER conducted, but advice incurred lower } \\
\text { costs and was more effective in proving daily } \\
\text { symptoms, pain bothersomeness, satisfaction, } \\
\text { days on sick leave (i.e. dominant). }\end{array}$ \\
\hline $\begin{array}{l}\text { Molde Hagen et al. } \\
\text { [27] }\end{array}$ & $\begin{array}{l}\text { Advice (advice and simple } \\
\text { exercises) } \\
\text { Usual care in primary care }\end{array}$ & $\begin{array}{l}\text { Type: CBA } \\
\text { Perspective: societal } \\
\text { Setting: Norway, price year } \\
1995 \\
\text { Follow-up: } 1 \text { year for costs, } \\
3 \text { years for other } \\
\text { outcomes }\end{array}$ & $\begin{array}{l}\text { Mean cost benefit }=3,497 \text { (1995 USD) favouring } \\
\text { advice }\end{array}$ \\
\hline $\begin{array}{l}\text { Niemisto et al. [23, } \\
\text { 24] }\end{array}$ & $\begin{array}{l}\text { Advice (advice, education and } \\
\text { simple exercises) } \\
\text { Advice plus manipulation and } \\
\text { stabilizing exercises }\end{array}$ & $\begin{array}{l}\text { Type: CEA/CUA } \\
\text { Perspective: societal } \\
\text { Setting: Finland, study } \\
\text { initiated in } 1999 \\
\text { Follow-up: } 2 \text { years }\end{array}$ & $\begin{array}{l}\text { ICER for advice plus manipulation and stabilizing } \\
\text { exercises compared to advice [mean (95\% CI)]: } \\
\text { Pain }=512 \text { (2002 USD) per } 1 \text { point gained on a } \\
\text { 100-point scale (77-949) } \\
\text { Disability (Oswestry) }=-78 \text { (2002 USD) per } 1 \\
\text { point gained on a } 100 \text {-point scale ( }-655 \text { to } 499)\end{array}$ \\
\hline $\begin{array}{l}\text { Rivero-Arias et al. } \\
\text { [38] }\end{array}$ & $\begin{array}{l}\text { Advice } \\
\text { Physiotherapy }\end{array}$ & $\begin{array}{l}\text { Type: CEA/CUA } \\
\text { Perspective: unspecified for } \\
\text { ICER } \\
\text { Setting: United Kingdom, } \\
\text { 1997-2001 } \\
\text { Follow-up: } 1 \text { year }\end{array}$ & $\begin{array}{l}\text { ICER for physiotherapy compared to advice: } \\
3,010 \text { (2004 GBP) per QALY gained (EQ-5D) }\end{array}$ \\
\hline $\begin{array}{l}\text { Strong et al. [20] for } \\
\text { Moore et al. [18] }\end{array}$ & $\begin{array}{l}\text { Book on back pain care } \\
\text { Advice (psychologist-led group } \\
\text { education sessions) }\end{array}$ & $\begin{array}{l}\text { Type: CEA/CUA } \\
\text { Perspective: health insurer } \\
\text { Setting: United States, } \\
\text { 1996-1997 } \\
\text { Follow-up: } 1 \text { year }\end{array}$ & $\begin{array}{l}\text { ICER for advice compared to book } \\
\text { [mean }(95 \% \mathrm{CI})] \text { : } \\
6.13 \text { (USD, price year not reported) per one } \\
\text { low-impact back pain days }(1.48-21.14)\end{array}$ \\
\hline
\end{tabular}


Table 2 continued

\begin{tabular}{|c|c|c|c|}
\hline Study ID & Comparative treatments & $\begin{array}{l}\text { Details of economic } \\
\text { evaluation }\end{array}$ & Results of economic evaluation \\
\hline $\begin{array}{l}\text { Strong et al. [20] for } \\
\text { von Korff et al. [19] }\end{array}$ & $\begin{array}{l}\text { Book on back pain care } \\
\text { Advice (layperson-led group } \\
\text { education sessions) }\end{array}$ & $\begin{array}{l}\text { Type: CEA/CUA } \\
\text { Perspective: health insurer } \\
\text { Setting: United States, } \\
\text { 1996-1997 } \\
\text { Follow-up: } 1 \text { year }\end{array}$ & $\begin{array}{l}\text { ICER for advice compared to book [mean }(95 \% \\
\text { CI)]: } \\
9.70 \text { (USD, price year not reported) per one low- } \\
\text { impact back pain days }(-45.45 \text { to } 78.86)\end{array}$ \\
\hline
\end{tabular}

The study included other comparison groups. Only data from the specified groups were included

$C B A$ cost-benefit analysis, $C E A$ cost-effectiveness analysis, $C U A$ cost-utility analysis, $G B P$ British pounds, GP care care provided by a general practitioner or a primary care physician, ICER incremental cost-effectiveness ratio, in cost per 1 unit of effect gained, $L B P$ low back pain, $Q A L Y$ quality-adjusted life-years, USD United States dollars

For patients who do not improve, consider the addition of spinal manipulation for acute LBP

Only one study investigated the cost-effectiveness of spinal manipulation in people with acute LBP [31]. Results of this cost-minimization study showed that while spinal manipulation was not the cheapest treatment option, the differences in costs over 1 year compared to care provided by a general practitioner (GP care) or exercise appeared small (total cost in Swedish crowns, price year not stated, for spinal manipulation $=49,076$, GP care $=50,834$ and exercise $=45,423$ ). However, there was no formal statistical comparison and this study had incomplete cost identification (only the costs of study treatment, investigations and operations were collected as the direct costs).

For patients who do not improve, consider the addition of interdisciplinary rehabilitation, exercise,

acupuncture, massage, spinal manipulation, yoga, cognitive-behavioural therapy or relaxation for sub-acute or chronic LBP

Fifteen studies investigated the cost-effectiveness of interdisciplinary rehabilitation, exercise, spinal manipulation or cognitive-behavioural therapy. These interventions were compared to conventional physiotherapy [29, 32, 33, 43], GP care [25, 28, 30, 35], spinal surgery [29] or walking [39], or as an additional treatment to advice [23, 24, 26], GP care [34, 36, 40] or inpatient rehabilitation [44]. Regardless of the comparisons and the perspectives adopted, all but two studies [30, 32] found that these interventions were cost-effective compared to the treatment alternatives (Table 3). In particular, Schweikert et al. [44] found that adding cognitive-behavioural therapy to inpatient rehabilitation dominated over inpatient rehabilitation alone $(\mathrm{ICER}=-126,731$ in 2001 Euro per QALY gained), and Torstensen et al. [29] found that medical exercise therapy dominated over walking (cost benefit $=906,732$ in Norwegian Kroner, price year not stated).
However, Schweikert et al. [44] had a follow-up of 6 months, which may be too short to adequately measure the economic consequences of chronic LBP. Torstensen et al. [29] did not specify the perspective of the economic evaluation. Given that people in the walking group incurred no costs, it is likely that this study conducted the economic evaluation from a narrow perspective, including the costs of the study treatment only.

Four studies compared interdisciplinary rehabilitation, exercise, spinal manipulation and cognitive-behavioural therapy to each other. Critchley et al. [33] found that a pain management programme using cognitive-behavioural principles was likely to be more cost-effective compared to exercise from the healthcare sector's perspective. Smeets et al. [42] found that, from a societal perspective, the combination of exercise with graded activity and problem solving was not cost-effective compared to exercise or graded activity and problem solving alone (e.g. ICER for exercise compared to combined group $=35,060$ in 2003 Euro per QALY gained, ICER for graded activity and problem solving compared to combined group $=$ dominant). In contrast, combining both manipulation and exercise with GP care was relatively cost-effective (ICER = 3,800 per QALY gained) compared to manipulation plus GP care (ICER $=4,800$ per QALY gained), or exercise plus GP care (ICER $=8,300$ per QALY gained) from the UK healthcare sector's perspective (in 2000-2001 GBP) [40]. One study showed that receiving an operant conditioning programme with group discussion or a cognitive behavioural component and relaxation incurred lower direct and indirect costs compared to waiting list then receiving the operant conditioning programme, but did not report an incremental cost-effectiveness analysis [41].

Witt et al. found that adding acupuncture to physician care had a low ICER compared to physician care alone from the societal perspective ( $€ 10,526$ per QALY gained in 2006 Euros, or GBP 7839.29 converted using purchasing power parities www.oecd.org/dataoecd/61/54/18598754. pdf) [45]. But this result is limited by methodological 
Table 3 The cost-effectiveness of interdisciplinary rehabilitation, exercise, spinal manipulation and cognitive-behavioural therapy (CBT) for sub-acute or chronic low back pain

\begin{tabular}{|c|c|c|c|}
\hline Study & Comparative treatments & Details of economic evaluation & Results of economic evaluation \\
\hline Critchley et al. [33] & $\begin{array}{l}\text { Physiotherapy } \\
\text { Spinal stabilization exercises } \\
\text { Pain management programme } \\
\text { using CBT }\end{array}$ & $\begin{array}{l}\text { Type: CEA/CUA } \\
\text { Perspective: healthcare sector } \\
\text { Setting: United Kingdom, 2002-2005 } \\
\text { Follow-up: } 18 \text { months }\end{array}$ & $\begin{array}{l}\text { No raw data reported, but pain } \\
\text { management programme associated } \\
\text { with least costs and acceptability curve } \\
\text { shows that it is likely to be most cost- } \\
\text { effective }\end{array}$ \\
\hline Hlobil et al. [26] & $\begin{array}{l}\text { Advice } \\
\text { Advice plus graded activity } \\
\text { using CBT }\end{array}$ & $\begin{array}{l}\text { Type: CBA } \\
\text { Perspective: employer } \\
\text { Setting: The Netherlands, 1999-2000 } \\
\text { Follow-up: } 1 \text { year for costs, } 3 \text { years } \\
\text { for other outcomes }\end{array}$ & $\begin{array}{l}\text { Mean cost benefit }=999 \text { (1999 Euro) } \\
\text { favouring advice plus graded activity } \\
(95 \% \text { CI }-1,073 \text { to } 3,115)\end{array}$ \\
\hline Hollinghurst et al. [34] & $\begin{array}{l}\text { GP care } \\
\text { GP care plus exercise and } \\
\text { behavioural counselling }\end{array}$ & $\begin{array}{l}\text { Type: CEA/CUA } \\
\text { Perspective: healthcare sector, } \\
\text { patients, societal } \\
\text { Setting: United Kingdom 2002-2004 } \\
\text { Follow-up: } 18 \text { months }\end{array}$ & $\begin{array}{l}\text { ICER for GP care plus exercise and } \\
\text { behavioural counselling compared to } \\
\text { GP care (in } 2005 \text { GBP per } 1 \text { unit of } \\
\text { effect gained, from the healthcare } \\
\text { sector's perspective only): } \\
\text { Disability (RMDQ) }=61 \\
\text { Pain-free days }=9 \\
\text { QALY gained (EQ-5D) }=2,847\end{array}$ \\
\hline Johnson et al. [35] & $\begin{array}{l}\text { GP care } \\
\text { Exercise and education using } \\
\text { CBT }\end{array}$ & $\begin{array}{l}\text { Type: CEA/CUA } \\
\text { Perspective: not stated } \\
\text { Setting: United Kingdom 2002-2003 } \\
\text { Follow-up: } 15 \text { months }\end{array}$ & $\begin{array}{l}\text { ICER for exercise and education using } \\
\text { CBT compared to GP care: } \\
5,000 \text { (2003-2004 GBP) per QALY } \\
\text { gained (EQ-5D) }\end{array}$ \\
\hline Kominski et al. [30] & $\begin{array}{l}\text { GP care } \\
\text { Chiropractic care } \\
\text { (manipulation, instruction in } \\
\text { back care and exercise) }\end{array}$ & $\begin{array}{l}\text { Type: CMA } \\
\text { Perspective: not stated } \\
\text { Setting: United States 1995-1998 } \\
\text { Follow-up: } 18 \text { months }\end{array}$ & $\begin{array}{l}\text { Costs over } 18 \text { months in USD [price year } \\
\text { not stated, mean }(\mathrm{SD})] \text { : } \\
\text { GP care }=463(1,225) \\
\text { Chiropractic care }=550(834) \\
\text { GP care significantly cheaper }\end{array}$ \\
\hline Lamb et al. [36] & $\begin{array}{l}\text { Group cognitive behavioural } \\
\text { intervention plus advice } \\
\text { Advice }\end{array}$ & $\begin{array}{l}\text { Type: CEA/CUA } \\
\text { Perspective: healthcare sector } \\
\text { Setting: United Kingdom, price year } \\
2008 \\
\text { Follow-up: } 1 \text { year }\end{array}$ & $\begin{array}{l}\text { ICER for Group cognitive behavioural } \\
\text { intervention plus advice }=1,786(2008 \\
\text { GBP) per QALY gained (EQ-5D) }\end{array}$ \\
\hline Loisel et al. [25] & $\begin{array}{l}\text { GP care } \\
\text { Clinical rehabilitation (back } \\
\text { pain specialist, back } \\
\text { school } \pm \text { multidisciplinary } \\
\text { rehabilitation) }\end{array}$ & $\begin{array}{l}\text { Type: CEA/CUA and CBA } \\
\text { Perspective: insurance provider } \\
\text { Setting: Canada 1991-1993 } \\
\text { Follow-up: mean } 6.4 \text { years }\end{array}$ & $\begin{array}{l}\text { ICER for treatments compared to GP care } \\
\text { (in } 1998 \text { Canadian dollars per } 1 \text { day on } \\
\text { full benefit): } \\
\text { Clinical rehabilitation }=-67.6 \\
\text { dominant }\end{array}$ \\
\hline Niemisto et al. $[23,24]$ & $\begin{array}{l}\text { Advice (advice, education and } \\
\text { simple exercises) } \\
\text { Advice plus manipulation and } \\
\text { stabilizing exercises }\end{array}$ & $\begin{array}{l}\text { Type: CEA/CUA } \\
\text { Perspective: societal } \\
\text { Setting: Finland, study initiated in } \\
1999 \\
\text { Follow-up: } 2 \text { years }\end{array}$ & $\begin{array}{l}\text { ICER for advice plus manipulation and } \\
\text { stabilizing exercises compared to advice } \\
\text { [in } 2002 \text { USD per } 1 \text { point gained, mean } \\
(95 \% \text { CI)]: } \\
\text { Pain }(0-100)=512(77-949) \\
\text { Disability }(\text { Oswestry, } 0-100)=-78 \\
(-655 \text { to } 499)\end{array}$ \\
\hline Rivero-Arias et al. [39] & $\begin{array}{l}\text { Outpatient rehabilitation } \\
\text { Spinal surgery }\end{array}$ & $\begin{array}{l}\text { Type: CEA/CUA } \\
\text { Perspective: healthcare sector and } \\
\text { patient } \\
\text { Setting: United Kingdom, 1996-2002 } \\
\text { Follow-up: } 2 \text { years }\end{array}$ & $\begin{array}{l}\text { ICER for spinal surgery compared to } \\
\text { outpatient rehabilitation [in } 2002-2003 \\
\text { GBP]: } \\
48,588 \text { per QALY gained } \\
\text { (95\% CI }-279,883 \text { to } 372,406)\end{array}$ \\
\hline
\end{tabular}


Table 3 continued

\begin{tabular}{|c|c|c|c|}
\hline Study & Comparative treatments & Details of economic evaluation & Results of economic evaluation \\
\hline Schweikert et al. [44] & $\begin{array}{l}\text { Inpatient rehabilitation } \\
\text { Inpatient rehabilitation plus } \\
\text { CBT }\end{array}$ & $\begin{array}{l}\text { Type: CEA/CUA } \\
\text { Perspective: societal } \\
\text { Setting: Germany, price year } 2001 \\
\text { Follow-up: } 6 \text { months }\end{array}$ & $\begin{array}{l}\text { ICER for inpatient rehabilitation plus } \\
\text { CBT compared to inpatient } \\
\text { rehabilitation } \\
\text {-126,731 (2001 Euro) per QALY gained } \\
\text { (EQ-5D, dominant) }\end{array}$ \\
\hline Skouen et al. [28] & $\begin{array}{l}\text { GP care } \\
\text { Light interdisciplinary } \\
\text { rehabilitation } \\
\text { Extensive interdisciplinary } \\
\text { rehabilitation }\end{array}$ & $\begin{array}{l}\text { Type: CBA } \\
\text { Perspective: societal } \\
\text { Setting: Norway 1996-1997 } \\
\text { Follow-up: } 2 \text { years after end of } \\
\text { treatment }\end{array}$ & $\begin{array}{l}\text { Cost benefit for treatments compared to } \\
\text { GP care: } \\
\text { Light interdisciplinary rehabilitation in } \\
\text { male patients }=7,240,900 \text { ( } 1998 \\
\text { Norwegian kroner) for the male } \\
\text { participants }(n=21) \text { over } 24 \text { months } \\
\text { Extensive interdisciplinary rehabilitation } \\
\text { - no data reported }\end{array}$ \\
\hline Torstensen et al. [29] & $\begin{array}{l}\text { Medical exercise therapy } \\
\text { Physiotherapy } \\
\text { Walking }\end{array}$ & $\begin{array}{l}\text { Type: CBA } \\
\text { Perspective: not reported } \\
\text { Setting: Norway, 1993-1996 } \\
\text { Follow-up: } 15 \text { months }\end{array}$ & $\begin{array}{l}\text { Cost benefit compared to walking in } \\
\text { Norwegian Kroner (price year not } \\
\text { reported): } \\
\text { Medical exercise therapy } \\
(n=69)=906,732 \text { less } \\
\text { Physiotherapy }(n=67)=1,882,560 \\
\text { less }\end{array}$ \\
\hline $\begin{array}{l}\text { UK BEAM Trial Team } \\
\text { [40] }\end{array}$ & $\begin{array}{l}\text { GP care } \\
\text { GP care plus exercise } \\
\text { GP care plus manipulation } \\
\text { GP care plus manipulation } \\
\text { followed by exercise }\end{array}$ & $\begin{array}{l}\text { Type: CEA/CUA } \\
\text { Perspective: healthcare sector } \\
\text { Setting: United Kingdom, 1999-2002 } \\
\text { Follow-up: } 1 \text { year }\end{array}$ & $\begin{array}{l}\text { ICER for treatments compared to GP care } \\
\text { [in 2000-2001 GBP per QALY gained } \\
\text { (EQ-5D)]: } \\
\text { GP care plus exercise }=8,300 \\
\text { GP care plus manipulation }=4,800 \\
\text { GP care plus manipulation followed by } \\
\text { exercise }=3,800\end{array}$ \\
\hline Van der Roer et al. [43] & $\begin{array}{l}\text { Exercise and back school } \\
\text { (using behavioural } \\
\text { principles) } \\
\text { Physiotherapy }\end{array}$ & $\begin{array}{l}\text { Type: CEA/CUA } \\
\text { Perspective: societal } \\
\text { Setting: The Netherlands, price year } \\
2004 \\
\text { Follow-up: } 1 \text { year }\end{array}$ & $\begin{array}{l}\text { ICER for exercise and back school } \\
\text { compared to physiotherapy (in } 2004 \\
\text { Euro per unit of effect gained): } \\
\text { Disability (RMDQ) }=16,349 \\
\text { Pain (numerical rating scale) }=-175 \\
\text { (dominant) } \\
\text { Perceived effects (Global perceived } \\
\text { effects scale) }=1,720 \\
\text { QALY }(E Q-5 D)=5,141\end{array}$ \\
\hline Whitehurst et al. [32] & $\begin{array}{l}\text { Physiotherapy } \\
\text { Pain management programme } \\
\text { using CBT }\end{array}$ & $\begin{array}{l}\text { Type: CEA/CUA } \\
\text { Perspective: healthcare sector } \\
\text { Setting: United Kingdom, price years } \\
\text { 2001-2002 } \\
\text { Follow-up: } 1 \text { year }\end{array}$ & $\begin{array}{l}\text { ICER for physiotherapy compared to pain } \\
\text { management (in 2001-2002 GBP per } \\
\text { unit of effect gained): } \\
\text { Disability (RMDQ) }=156 \\
\text { QALY (EQ-5D) }=2,362\end{array}$ \\
\hline
\end{tabular}

$\overline{C B A}$ cost-benefit analysis, $C E A$ cost-effectiveness analysis, $C U A$ cost-utility analysis, GBP British pounds, GP care care provided by a general practitioner or a primary care physician, ICER incremental cost-effectiveness ratio, $L B P$ low back pain, $Q A L Y$ quality-adjusted life-years, $U S D$ United States dollars

issues. For example, the costs collected (provided by health insurance funds) may be incomplete for the perspective adopted (societal), the methods of cost valuation were not reported, and data were collected immediately after the treatment period with no other follow-up. Ratcliffe et al. [37] showed that acupuncture had a low ICER compared to GP care from the healthcare sector perspective (4,241 in 2002 to 2003 GBP per QALY gained, 95\%
CI $=191-28,026)$, and was dominant over GP care from the societal perspective.

Hollinghurst et al. [34] investigated the cost-effectiveness of massage over a 1-year period. Compared to GP care, massage incurred higher costs from the healthcare sector's perspective and was less effective (QALY gained = $-34,473$ in 2005 GBP). But adding exercise and behavioural counselling to massage improved the cost-effectiveness of 
massage (ICER compared to GP care plus exercise and behavioural counselling $=5,304$ in 2005 GBP). No studies investigated the cost-effectiveness of yoga or relaxation.

\section{Discussion}

We found 26 economic evaluations conducted alongside randomised controlled trials that investigated the costeffectiveness of guideline-endorsed treatments for nonspecific LBP. There were inconsistent findings regarding the cost-effectiveness of advice, but studies generally showed that interdisciplinary rehabilitation, exercise, acupuncture, spinal manipulation and cognitive-behavioural therapy were relatively cost-effective for people with subacute or chronic LBP. Results from single studies suggested that massage alone was unlikely to be cost-effective, and that the cost-effectiveness of spinal manipulation for acute LBP was unclear. No studies investigated the costeffectiveness of medication, yoga or relaxation as treatments for LBP.

The eight studies which investigated advice did not yield consistent or conclusive evidence about its relative costeffectiveness. Interestingly, these studies also reported inconsistencies in the effectiveness of advice compared to other treatments. In contrast, the American clinical guideline made a strong recommendation for advice to stay active based on moderate-quality evidence [3]. Other guidelines also recommend advice to stay active [5]. One reason for the difference between our findings and guideline recommendations may be that in the guidelines, the recommendations were based on evidence from systematic reviews which compared advice to stay active with bed rest, which is considered potentially harmful for this population [47]. The studies included in this review compared advice to a variety of treatment alternatives, but not bed rest.

We did not pool the results in the studies that compared advice, and interdisciplinary rehabilitation, exercise, acupuncture, spinal manipulation and cognitive-behavioural therapy for sub-acute or chronic LBP, as may occur in a systematic review of treatment effectiveness. This is due to the heterogeneity in study treatments, as well as differences in economic perspectives and settings. The underlying assumption for pooling in a systematic review of treatment effectiveness is that results obtained in one country are generalisable to a similar population in a different setting or country. Whilst it seems reasonable to assume that individuals or groups are likely to react in the same way to a particular intervention, no matter where they live, comparing economic data across different settings or countries is not as straightforward due to differences in the structure and organization of healthcare systems. For example, in some countries patients may have direct access to medical specialists or other healthcare providers while in other countries patients need a referral from a primary care physician. Access to some care providers may be limited in some countries where this care is not provided by a public healthcare system or is not reimbursed by an insurance scheme. Cost data may also be sensitive to the funding and reimbursement arrangements in a particular healthcare system. However, despite this complexity, there are emerging guidelines on the transferability of economic evaluations [48-50].

We used the NICE threshold to provide an indication of the cost-effectiveness of treatment, because the NICE threshold is commonly available. However, it should be noted that there is no consensus about the maximum costs per QALY gained that would be acceptable, and recent evidence indicates that the cost-effectiveness threshold may vary depending upon the severity and the prevalence of the disease. We used the treatments endorsed by the American College of Physicians and the American Pain Society as guideline-endorsed treatments, because at the time of study conception the American guideline was one of the most recent guidelines. A recent review shows that treatments endorsed by the American guideline are in line with other guidelines [5]. The only area of contention is in the use of spinal manipulation where, unlike the American guideline, some countries do not recommend spinal manipulation for LBP. Interestingly, our systematic review considers evidence purely from a cost-effectiveness perspective and shows some evidence of cost-effectiveness when using spinal manipulation in sub-acute to chronic pain.

There were some methodological issues which limit the interpretation of our findings. These include the incomplete identification and measurement of costs, which reduces the rigour of the results. Three studies had follow-up periods that are likely to be too short to fully appreciate the economic consequences for the chronic population under investigation [44-46]. Based on recent large cohort studies on the prognosis of acute [51] and chronic [52] LBP, we recommend a follow-up period of at least 3 months for acute LBP and at least 12 months for chronic LBP. In addition, to help readers assess the extent to which the results of studies are applicable to different healthcare systems, we recommend that economic evaluations report unit costs as well as reporting a breakdown of costs and resource utilization. Eleven of the 26 included studies provided a table of unit costs $[21,22,33,34$, 37-40, 42, 43, 46, 53].

Considering the evidence regarding both relative effectiveness and cost-effectiveness when making treatment recommendations means that the endorsed treatments are both beneficial to patients and efficient in terms of healthcare resources. The small number or lack of economic evaluations for some guideline-endorsed treatments means well- 
conducted economic evaluations are required to strengthen the evidence-base of treatments for LBP. However, evidence to date indicates that guideline-endorsed treatments such as interdisciplinary rehabilitation, exercise, acupuncture, spinal manipulation and cognitive-behavioural therapy for sub-acute or chronic LBP are cost-effective. Although advice to stay active is endorsed in the guideline, and evidence regarding its cost-effectiveness compared to other interventions is inconsistent. In addition, there is little or no high-level evidence about other guideline-endorsed treatments: medication, spinal manipulation for acute LBP, and massage, yoga or relaxation for chronic LBP.

Acknowledgments $\mathrm{CL}$ and $\mathrm{CM}$ are funded by the National Health and Medical Research Council, Australia. LM is funded by Fundação de Amparo à Pesquisa do Estado de Minas Gerais (FAPEMIG), Brazil.

Open Access This article is distributed under the terms of the Creative Commons Attribution Noncommercial License which permits any noncommercial use, distribution, and reproduction in any medium, provided the original author(s) and source are credited.

\section{Appendix: Search strategy for Embase (via OvidSP)}

Part A: Generic search for randomized controlled trials and controlled clinical trials

1 Clinical Article/

2 exp Clinical Study/

3 Clinical Trial/

4 Controlled Study/

5 Randomized Controlled Trial/

6 Major Clinical Study/

7 Double Blind Procedure/

8 Multicenter Study/

9 Single Blind Procedure/

10 Phase 3 Clinical Trial/

11 Phase 4 Clinical Trial/

12 crossover procedure/

13 placebo/

14 or/1-13

15 allocat\$.mp.

16 assign\$.mp.

17 blind\$.mp.

18 (clinic\$ adj25 (study or trial)).mp.

19 compar\$.mp.

20 control\$.mp.

21 cross?over.mp.

22 factorial\$.mp.
Appendix continued

23 follow?up.mp.

24 placebo\$.mp.

25 prospectiv\$.mp.

26 random\$.mp.

27 ((singl\$ or doubl\$ or trebl\$ or tripl\$) adj25 (blind\$ or mask\$)).mp.

28 trial.mp.

29 (versus or vs).mp.

30 or/15-29

$31 \quad 14$ and 30

32 human/

33 Nonhuman/

34 exp ANIMAL/

35 Animal Experiment/

3633 or 34 or 35

$37 \quad 32$ not 36

3831 not 36

$39 \quad 37$ and 38

4038 or 39

Part B: Specific search for low back problems

Part C: Specific search for economic evaluation

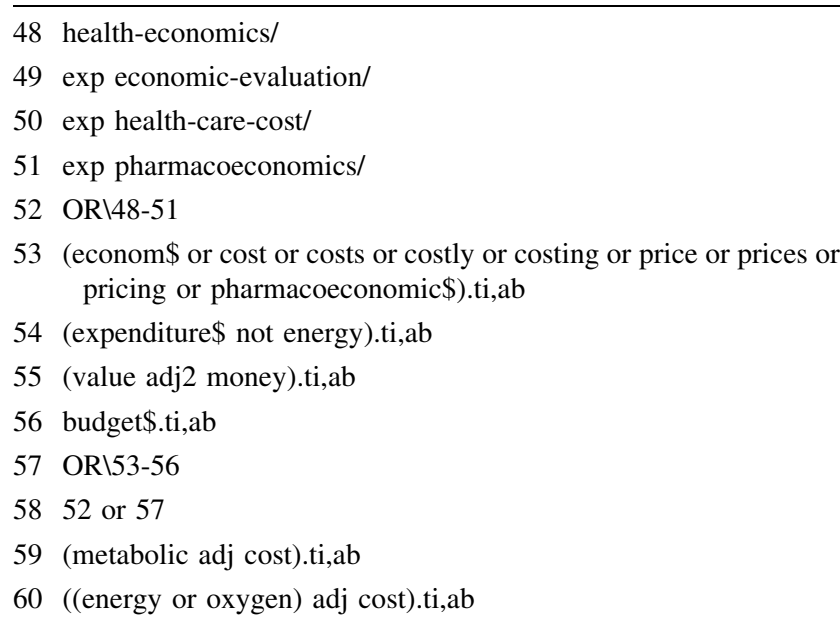

exp pharmacoeconomics/

OR\48-5

pricing or pharmacoeconomic\$).ti,ab

55 (value adj2 money).ti,ab

56 budget $\$ . t i, a b$

57 OR $153-56$

59 (metabolic adj cost).ti,ab 


\section{Appendix continued}

61 ((energy or oxygen) near expenditure).ti,ab

62 OR\59-61

6358 not 62

Part D: combine

$64 \quad 40$ AND 47 AND 63

\begin{tabular}{ll}
\hline Included studies & Related publications used \\
\hline - Critchley DJ, Ratcliffe J, Nil \\
Noonan S, Jones RH, Hurley \\
MV (2007) Effectiveness and \\
cost-effectiveness of three types \\
of physiotherapy used to reduce \\
chronic low back pain \\
disability: a pragmatic \\
randomized trial with economic \\
evaluation. Spine \\
32(14):1474-1481 \\
- Goossens ME, Rutten-Van \\
Molken MP, Kole-Snijders AM, \\
Vlaeyen JW, Van Breukelen G, \\
Leidl R (1998) Health economic \\
assessment of behavioural \\
rehabilitation in chronic low \\
back pain: a randomised clinical \\
trial. Health Econ 7(1):39-51
\end{tabular}

- Herman PM, Szczurko O, Cooley K, Mills EJ (2008) Costeffectiveness of naturopathic care for chronic low back pain. Altern Ther Health Med 14(2):32-39

- Szczurko O, Cooley K, Busse JW, Seely D, Bernhardt B, Guyatt GH, et al (2007) Naturopathic care for chronic low back pain: a randomized trial. PLoS One 2:e919

- Hlobil H, Uegaki K, Staal JB, de Bruyne MC, Smid T, van Mechelen W (2007) Substantial sick-leave costs savings due to a graded activity intervention for workers with non-specific subacute low back pain. Eur Spine J 16(7):919-924

- Hollinghurst S, Sharp D, Ballard K, Barnett J, Beattie A, Evans M, Lewith G, Middleton K, Oxford F, Webley F, Little P (2008) Randomised controlled trial of alexander technique lessons, exercise, and massage (ateam) for chronic and recurrent back pain: economic evaluation. BMJ 337:a2656
- Staal JB, Hlobil H, Twisk JW, Smid T, Köke AJ, van Mechelen W (2004) Graded activity for low back pain in occupational health care: a randomized, controlled trial. Ann Intern Med 140:77-84

- Little P, Lewith G, Webley F, Evans M, Beattie A, Middleton $\mathrm{K}$, et al (2008) Randomised controlled trial of Alexander technique lessons, exercise, and massage (ATEAM) for chronic and recurrent back pain. BMJ 337:a884
Appendix continued

\begin{tabular}{|c|c|}
\hline Included studies & Related publications used \\
\hline $\begin{array}{l}\text { - Johnson RE, Jones GT, Wiles } \\
\text { NJ, Chaddock C, Potter RG, } \\
\text { Roberts C, Symmons DPM, } \\
\text { Watson PJ, Torgerson DJ, } \\
\text { Macfarlane GJ (2007) Active } \\
\text { exercise, education, and } \\
\text { cognitive behavioral therapy for } \\
\text { persistent disabling low back } \\
\text { pain: a randomized controlled } \\
\text { trial. Spine 32(15):1578-1585 }\end{array}$ & Nil \\
\hline $\begin{array}{l}\text { Karjalainen K, Malmivaara A, } \\
\text { Mutanen P, Roine R, Hurri H, } \\
\text { Pohjolainen T (2004) Mini- } \\
\text { intervention for subacute low } \\
\text { back pain: two-year follow-up } \\
\text { and modifiers of effectiveness. } \\
\text { Spine 29(10):1069-1076 }\end{array}$ & Nil \\
\hline $\begin{array}{l}\text { - Karjalainen K, Malmivaara A, } \\
\text { Pohjolainen T, Hurri H, } \\
\text { Mutanen P, Rissanen P, } \\
\text { Pahkajarvi H, Levon H, Karpoff } \\
\text { H, Roine R (2003) Mini- } \\
\text { intervention for subacute low } \\
\text { back pain: a randomized } \\
\text { controlled trial. Spine } \\
\text { 28(6):533-540 }\end{array}$ & \\
\hline $\begin{array}{l}\text { Kominski GF, Heslin KC, } \\
\text { Morgenstern H, Hurwitz EL, } \\
\text { Harber PI (2005) Economic } \\
\text { evaluation of four treatments for } \\
\text { low-back pain: results from a } \\
\text { randomized controlled trial. } \\
\text { Med Care 43(5):428-435 }\end{array}$ & $\begin{array}{l}\text { - Hurwitz EL, Morgenstern H, } \\
\text { Harber P, Kominski GF, Belin } \\
\text { TR, Yu F, et al (2002) A } \\
\text { randomized trial of medical care } \\
\text { with and without physical } \\
\text { therapy and chiropractic care } \\
\text { with and without physical } \\
\text { modalities for patients with low } \\
\text { back pain: 6-month follow-up } \\
\text { outcomes from the UCLA low } \\
\text { back pain study. Spine } \\
\text { 27:2193-2204 }\end{array}$ \\
\hline $\begin{array}{l}\text { Lamb SE, Hansen Z, Lall R, } \\
\text { Castelnuovo E, Withers EJ, } \\
\text { Nichols V, Potter R, } \\
\text { Underwood MR (2010) Group } \\
\text { cognitive behavioural treatment } \\
\text { for low-back pain in primary } \\
\text { care: a randomised controlled } \\
\text { trial and cost-effectiveness } \\
\text { analysis. Lancet } \\
\text { 375(9718):916-923 }\end{array}$ & Nil \\
\hline $\begin{array}{l}\text { Loisel P, Lemaire J, Poitras S, } \\
\text { Durand MJ, Champagne F, } \\
\text { Stock S, Diallo B, Tremblay C } \\
\text { (2002) Cost-benefit and cost- } \\
\text { effectiveness analysis of a } \\
\text { disability prevention model for } \\
\text { back pain management: a six } \\
\text { year follow up study. Occup } \\
\text { Environ Med 59(12):807-815 }\end{array}$ & $\begin{array}{l}\text { - Loisel P, Abenhaim L, Durand } \\
\text { P, Esdaile JM, Suissa S, } \\
\text { Gosselin L, et al (1997) A } \\
\text { population-based, randomized } \\
\text { clinical trial on back pain } \\
\text { management. Spine } \\
\text { 22:2911-2918 }\end{array}$ \\
\hline
\end{tabular}

Johnson RE, Jones GT, Wile Roberts C, Symmons DPM, Watson PJ, Torgerson DJ, exercise, education, and pain: a randomized controlle Mutanen P, Roine R, Hurri H, Karjalainen K, Malmivaara A, Pohjolainen T, Hurri H, Mutanen P, Rissanen $\mathrm{P}$ kajarvi H, Levon H, Karpoff

Kominski GF, Heslin KC, Morgenstern H, Hurwitz EL, randomized controlled trial. 西 
Appendix continued

\begin{tabular}{l}
\hline Included studies \\
\hline Molde Hagen E, Grasdal A, \\
Eriksen HR (2003) Does early \\
intervention with a light \\
mobilization program reduce \\
long-term sick leave for low \\
back pain: a 3-year follow-up \\
study. Spine 28(20):2309-2315 \\
- Niemisto L, Lahtinen-Suopanki \\
T, Rissanen P, Lindgren K-A, \\
Sarna S, Hurri H (2003) A \\
randomized trial of combined \\
manipulation, stabilizing \\
exercises, and physician \\
consultation compared to \\
physician consultation alone for \\
chronic low back pain. Spine \\
28(19):2185-2191 \\
- Niemisto L, Rissanen P, Sarna \\
S, Lahtinen-Suopanki T, \\
Lindgren K-A, Hurri H (2005) \\
Cost-effectiveness of combined \\
manipulation, stabilizing \\
exercises, and physician \\
consultation compared to \\
physician consultation alone for \\
chronic low back pain: a \\
prospective randomized trial \\
with 2-year follow-up. Spine \\
30(10):1109-1115 \\
- Ratcliffe J, Thomas KJ, \\
\end{tabular}

MacPherson H, Brazier J (2006) A randomised controlled trial of acupuncture care for persistent low back pain: cost effectiveness analysis. BMJ 333(7569):626

- Rivero-Arias O, Gray A, Frost H, Lamb SE, Stewart-Brown S (2006) Cost-utility analysis of physiotherapy treatment compared with physiotherapy advice in low back pain. Spine 31(12):1381-1387

- Rivero-Arias O, Campbell H, Gray A, Fairbank J, Frost H, Wilson-MacDonald J (2005) Surgical stabilisation of the spine compared with a programme of intensive rehabilitation for the management of patients with chronic low back pain: cost utility analysis based on a randomised controlled trial. BMJ 330(7502):1239
Related publications used
Nil

.

$\bullet$

- Thomas KJ, MacPherson H, Ratcliffe J, Thorpe L, Brazier J, Campbell M, et al (2005) Longer term clinical and economic benefits of offering acupuncture care to patients with chronic low back pain. Health Technol Assess 9:iii-iv, ix-x, 1-109

- Frost H, Lamb SE, Doll HA, Carver PT, Stewart-Brown S (2004) Randomised controlled trial of physiotherapy compared with advice for low back pain. BMJ 329:708

- Fairbank J, Frost H, WilsonMacDonald J, Yu LM, Barker K, Collins R, et al (2005) Randomised controlled trial to compare surgical stabilisation of the lumbar spine with an intensive rehabilitation programme for patients with chronic low back pain: the MRC spine stabilisation trial. BMJ 330:1233 G (2000) Cost-minimisation analysis of three conservative treatment programmes in 180 patients sick-listed for acute low-back pain. Scand J Prim Health Care 18(1):53-57

- Skouen JS, Grasdal AL, Haldorsen EMH, Ursin H (2002) Relative costeffectiveness of extensive and light multidisciplinary treatment programs versus treatment as usual for patients with chronic low back pain on long-term sick leave: randomized controlled study. Spine 27(9):901-909

- Seferlis T, Németh G, Carlsson AM, Gillström P (1998) Conservative treatment in patients sick-listed for acute low-back pain: a prospective randomised study with 12 months' follow-up. Eur Spine J 7:461-470

- Haldorsen EM, Grasdal AL, Skouen JS, Risa AE, Kronholm $\mathrm{K}$, Ursin H (2002) Is there a right treatment for a particular patient group? Comparison of ordinary treatment, light multidisciplinary treatment, and extensive multidisciplinary treatment for long-term sicklisted employees with musculoskeletal pain. Pain 95:49-63

- Smeets RJ, Severens JL, Beelen Nil

S, Vlaeyen JW, Knottnerus J (2009) More is not always better: cost-effectiveness analysis of combined, single behavioral and single physical rehabilitation programs for chronic low back pain. Eur $\mathrm{J}$ Pain 13(1):71-81

- Strong LL, Von Korff M, Saunders K, Moore JE (2006) Cost-effectiveness of two selfcare interventions to reduce disability associated with back pain. Spine 31(15):1639-1645

- Moore JE, Von Korff M, Cherkin D, Saunders K, Lorig $\mathrm{K}$. A randomized trial of a cognitive-behavioral program for enhancing back pain self care in a primary care setting. Pain. 2000;88:145-153

- Von Korff M, Moore JE, Lorig K, Cherkin DC, Saunders K, González VM, et al (1998) A randomized trial of a lay person-led self-management group intervention for back pain patients in primary care. Spine 23:2608-2615 

kingdom back pain exercise and manipulation (UK BEAM) randomised trial: cost effectiveness of physical treatments for back pain in primary care. BMJ 329(7479):1381-1385
Related publications used
- Brealey S, Burton K, Coulton S, Farrin A, Garratt A, Harvey E, et al (2003) UK Back pain Exercise And Manipulation (UK BEAM) trial - national randomised trial of physical treatments for back pain in primary care: objectives, design and interventions [ISRCTN32683578]. BMC Health Serv Res 3:16

- UK BEAM Trial Team (2004) United Kingdom back pain exercise and manipulation (UK BEAM) randomised trial: effectiveness of physical treatments for back pain in primary care. BMJ 329:1377

- van der Roer N, van Tulder M, van Mechelen W, de Vet $\mathrm{H}$ (2008) Economic evaluation of an intensive group training protocol compared with usual care physiotherapy in patients with chronic low back pain. Spine 33(4):445-451

- Whitehurst DGT, Lewis M, Yao GL, Bryan S, Raftery JP, Mullis R, Hay EM (2007) A brief pain management program compared with physical therapy for low back pain: results from an economic analysis alongside a randomized clinical trial. Arthritis Rheum 57(3):466-473

- Witt CM, Jena S, Selim D, Brinkhaus B, Reinhold T, Wruck K, Liecker B, Linde K, Wegscheider K, Willich SN (2006) Pragmatic randomized trial evaluating the clinical and economic effectiveness of acupuncture for chronic low back pain. Am J Epidemiol 164(5):487-496

\section{References}

1. Hestbaek L, Leboeuf-Yde C, Manniche C (2003) Low back pain: what is the long-term course? A review of studies of general patient populations. Eur Spine J 12:149-165

2. Koes BW, van Tulder MW, Thomas S (2006) Diagnosis and treatment of low back pain. BMJ 332(7555):1430-1434

3. Chou R, Qaseem A, Snow V, Casey D, Cross JT Jr, Shekelle P, Owens DK, Clinical Efficacy Assessment Subcommittee of the American College of Physicians; American College of Physicians; American Pain Society Low Back Pain Guidelines Panel (2007) Diagnosis and treatment of low back pain: a joint clinical practice guideline from the American College of Physicians and the American Pain Society. Ann Intern Med 147(7):478-491

4. Henschke N, Maher CG, Refshauge KM, Herbert RD, Cumming RG, Bleasel J, York J, Das A, McAuley JH (2009) Prevalence of and screening for serious spinal pathology in patients presenting to primary care with acute low back pain. Arthritis Rheum 60(10):3072-3080

5. Koes B, van Tulder M, Lin C, Macedo L, McAuley JH, Maher C (Accepted 16 June 2010) An updated overview of clinical guidelines for the management of nonspecific low back pain in primary care. Eur Spine J 19(12):2075-2094

6. The cochrane library, issue 3 2009. http://www3.interscience. wiley.com/cgi-bin/mrwhome/106568753/HOME?CRETRY=1\& SRETRY=0. Accessed 22 September 2009

7. Savigny $\mathrm{P}$, Kuntze S, Watson P, Underwood M, Ritchie G, Cotterell M, Hill D, Browne N, Buchanan E, Coffey P, Dixon P, Drummond C, Flanagan M, Greenough C, Griffiths M, HallidayBell J, Hettinga D, Vogel S, Walsh D (2009) Low back pain: early management of persistent non-specific low back pain. National Collaborating Centre for Primary Care and Royal College of General Practitioners, London

8. van der Roer N, Goossens ME, Evers SM, van Tulder MW (2005) What is the most cost-effective treatment for patients with low back pain? A systematic review. Best Pract Res Clin Rheumatol 19(4):671-684

9. Dagenais S, Roffey DM, Wai EK, Haldeman S, Caro J (2009) Can cost utility evaluations inform decision making about interventions for low back pain? Spine J 9(11):944-957

10. Dagenais S, Caro J, Haldeman S (2008) A systematic review of low back pain cost of illness studies in the United States and internationally. Spine J 8(1):8-20

11. Martin BI, Deyo RA, Mirza SK, Turner JA, Comstock BA, Hollingworth W, Sullivan SD (2008) Expenditures and health status among adults with back and neck problems. JAMA 299(6):656-664

12. Drummond MF, Sculpher MJ, Torrance GW, O'Brien BJ, Stoddart GL (2005) Methods for the economic evaluation of health care programmes, 3rd edn. Oxford University Press, Oxford

13. Furlan AD, Pennick V, Bombardier C, van Tulder M, Editorial Board Cochrane Back Review Group (2009) 2009 updated method guidelines for systematic reviews in the cochrane back review group. Spine 34(18):1929-1941

14. van Tulder M, Furlan A, Bombardier C, Bouter L, Editorial Board of the Cochrane Collaboration Back Review Group (2003) Updated method guidelines for systematic reviews in the cochrane collaboration back review group. Spine 28(12):12901299

15. Evers S, Goossens M, de Vet H, van Tulder M, Ament A (2005) Criteria list for assessment of methodological quality of economic evaluations: Consensus on health economic criteria. Int $\mathbf{J}$ Technol Assess Health Care 21(2):240-245 
16. van Tulder M, Suttorp M, Morton S, Bouter L, Shekelle P (2009) Empirical evidence of an association between internal validity and effect size in randomized controlled trials of low-back pain. Spine 34(16):1685-1692

17. Appleby J, Devlin N, Parkin D (2007) Nice's cost effectiveness threshold. BMJ 335(7616):358-359

18. Moore JE, Von Korff M, Cherkin D, Saunders K, Lorig K (2000) A randomized trial of a cognitive-behavioral program for enhancing back pain self care in a primary care setting. Pain 88(2):145-153

19. Von Korff M, Moore JE, Lorig K, Cherkin DC, Saunders K, González VM, Laurent D, Rutter C, Comite F (1998) A randomized trial of a lay person-led self-management group intervention for back pain patients in primary care. Spine 23(23): $2608-2615$

20. Strong LL, Von Korff M, Saunders K, Moore JE (2006) Costeffectiveness of two self-care interventions to reduce disability associated with back pain. Spine 31(15):1639-1645

21. Karjalainen K, Malmivaara A, Mutanen P, Roine R, Hurri H, Pohjolainen T (2004) Mini-intervention for subacute low back pain: two-year follow-up and modifiers of effectiveness. Spine 29(10):1069-1076

22. Karjalainen K, Malmivaara A, Pohjolainen T, Hurri H, Mutanen $\mathrm{P}$, Rissanen $\mathrm{P}$, Pahkajarvi H, Levon $\mathrm{H}$, Karpoff $\mathrm{H}$, Roine $\mathrm{R}$ (2003) Mini-intervention for subacute low back pain: a randomized controlled trial. Spine 28(6):533-540

23. Niemisto L, Lahtinen-Suopanki T, Rissanen P, Lindgren K-A, Sarna S, Hurri H (2003) A randomized trial of combined manipulation, stabilizing exercises, and physician consultation compared to physician consultation alone for chronic low back pain. Spine 28(19):2185-2191

24. Niemisto L, Rissanen P, Sarna S, Lahtinen-Suopanki T, Lindgren K-A, Hurri H (2005) Cost-effectiveness of combined manipulation, stabilizing exercises, and physician consultation compared to physician consultation alone for chronic low back pain: a prospective randomized trial with 2-year follow-up. Spine 30(10):1109-1115

25. Loisel P, Lemaire J, Poitras S, Durand MJ, Champagne F, Stock S, Diallo B, Tremblay C (2002) Cost-benefit and cost-effectiveness analysis of a disability prevention model for back pain management: a six year follow up study. Occup Environ Med 59(12):807-815

26. Hlobil H, Uegaki K, Staal JB, de Bruyne MC, Smid T, van Mechelen W (2007) Substantial sick-leave costs savings due to a graded activity intervention for workers with non-specific subacute low back pain. Eur Spine J 16(7):919-924

27. Molde Hagen E, Grasdal A, Eriksen HR (2003) Does early intervention with a light mobilization program reduce long-term sick leave for low back pain: a 3-year follow-up study. Spine 28(20):2309-2315

28. Skouen JS, Grasdal AL, Haldorsen EMH, Ursin H (2002) Relative cost-effectiveness of extensive and light multidisciplinary treatment programs versus treatment as usual for patients with chronic low back pain on long-term sick leave: randomized controlled study. Spine 27(9):901-909

29. Torstensen TA, Ljunggren AE, Meen HD, Odland E, Mowinckel P, Geijerstam S (1998) Efficiency and costs of medical exercise therapy, conventional physiotherapy, and self-exercise in patients with chronic low back pain. A pragmatic, randomized, singleblinded, controlled trial with 1-year follow-up. Spine 23(23): 2616-2624

30. Kominski GF, Heslin KC, Morgenstern H, Hurwitz EL, Harber PI (2005) Economic evaluation of four treatments for low-back pain: results from a randomized controlled trial. Med Care 43(5):428-435
31. Seferlis T, Lindholm L, Nemeth G (2000) Cost-minimisation analysis of three conservative treatment programmes in 180 patients sick-listed for acute low-back pain. Scand J Prim Health Care 18(1):53-57

32. Whitehurst DGT, Lewis M, Yao GL, Bryan S, Raftery JP, Mullis R, Hay EM (2007) A brief pain management program compared with physical therapy for low back pain: results from an economic analysis alongside a randomized clinical trial. Arthritis Rheum 57(3):466-473

33. Critchley DJ, Ratcliffe J, Noonan S, Jones RH, Hurley MV (2007) Effectiveness and cost-effectiveness of three types of physiotherapy used to reduce chronic low back pain disability: a pragmatic randomized trial with economic evaluation. Spine 32(14):1474-1481

34. Hollinghurst S, Sharp D, Ballard K, Barnett J, Beattie A, Evans M, Lewith G, Middleton K, Oxford F, Webley F, Little P (2008) Randomised controlled trial of alexander technique lessons, exercise, and massage (ateam) for chronic and recurrent back pain: economic evaluation. BMJ 337:a2656

35. Johnson RE, Jones GT, Wiles NJ, Chaddock C, Potter RG, Roberts C, Symmons DPM, Watson PJ, Torgerson DJ, Macfarlane GJ (2007) Active exercise, education, and cognitive behavioral therapy for persistent disabling low back pain: a randomized controlled trial. Spine 32(15):1578-1585

36. Lamb SE, Hansen Z, Lall R, Castelnuovo E, Withers EJ, Nichols V, Potter R, Underwood MR (2010) Group cognitive behavioural treatment for low-back pain in primary care: a randomised controlled trial and cost-effectiveness analysis. Lancet 375(9718): 916-923

37. Ratcliffe J, Thomas KJ, MacPherson H, Brazier J (2006) A randomised controlled trial of acupuncture care for persistent low back pain: cost effectiveness analysis. BMJ 333(7569):626

38. Rivero-Arias O, Gray A, Frost H, Lamb SE, Stewart-Brown S (2006) Cost-utility analysis of physiotherapy treatment compared with physiotherapy advice in low back pain. Spine 31(12):13811387

39. Rivero-Arias O, Campbell H, Gray A, Fairbank J, Frost H, Wilson-MacDonald J (2005) Surgical stabilisation of the spine compared with a programme of intensive rehabilitation for the management of patients with chronic low back pain: cost utility analysis based on a randomised controlled trial. BMJ 330(7502): 1239

40. UK BEAM Trial (2004) United kingdom back pain exercise and manipulation (UK BEAM) randomised trial: cost effectiveness of physical treatments for back pain in primary care. BMJ 329(7479):1381-1385

41. Goossens ME, Rutten-Van Molken MP, Kole-Snijders AM, Vlaeyen JW, Van Breukelen G, Leidl R (1998) Health economic assessment of behavioural rehabilitation in chronic low back pain: a randomised clinical trial. Health Econ 7(1):39-51

42. Smeets RJ, Severens JL, Beelen S, Vlaeyen JW, Knottnerus J (2009) More is not always better: cost-effectiveness analysis of combined, single behavioral and single physical rehabilitation programs for chronic low back pain. Eur J Pain 13(1):71-81

43. van der Roer N, van Tulder M, van Mechelen W, de Vet H (2008) Economic evaluation of an intensive group training protocol compared with usual care physiotherapy in patients with chronic low back pain. Spine 33(4):445-451

44. Schweikert B, Jacobi E, Seitz R, Cziske R, Ehlert A, Knab J, Leidl R (2006) Effectiveness and cost-effectiveness of adding a cognitive behavioral treatment to the rehabilitation of chronic low back pain. J Rheumatol 33(12):2519-2526

45. Witt CM, Jena S, Selim D, Brinkhaus B, Reinhold T, Wruck K, Liecker B, Linde K, Wegscheider K, Willich SN (2006) Pragmatic randomized trial evaluating the clinical and economic 
effectiveness of acupuncture for chronic low back pain. Am J Epidemiol 164(5):487-496

46. Herman PM, Szczurko O, Cooley K, Mills EJ (2008) Costeffectiveness of naturopathic care for chronic low back pain. Altern Ther Health Med 14(2):32-39

47. Hilde G, Hagen KB, Jamtvedt G, Winnem M (2002) Advice to stay active as a single treatment for low back pain and sciatica. Cochrane Database Syst Rev (2):CD003632

48. Boulenger S, Nixon J, Drummond M, Ulmann P, Rice S, de Pouvourville G (2005) Can economic evaluations be made more transferable? Eur J Health Econ 6(4):334-346

49. Edejer TT (2006) Improving the use of research evidence in guideline development: 11. Incorporating considerations of costeffectiveness, affordability and resource implications. Health Res Policy Syst 4:23

50. Goeree R, Burke N, O'Reilly D, Manca A, Blackhouse G, Tarride JE (2007) Transferability of economic evaluations: approaches and factors to consider when using results from one geographic area for another. Curr Med Res Opin 23(4):671-682

51. Henschke N, Maher CG, Refshauge KM, Herbert RD, Cumming RG, Bleasel J, York J, Das A, McAuley JH (2008) Prognosis in patients with recent onset low back pain in Australian primary care: inception cohort study. BMJ 337:a171

52. Costa LdC, Maher CG, McAuley JH, Hancock MJ, Herbert RD, Refshauge KM, Henschke N (2009) Prognosis for patients with chronic low back pain: inception cohort study. BMJ 339:b3829

53. Jellema $\mathrm{P}$, van der Roer N, van der Windt DAWM, van Tulder MW, van der Horst HE, Stalman WAB, Bouter LM (2007) Low back pain in general practice: cost-effectiveness of a minimal psychosocial intervention versus usual care. Eur Spine J 16(11):1812-1821 